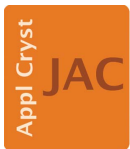

JOURNAL OF

APPLIED

CRYSTALLOGRAPHY

ISSN 1600-5767

Received 16 November 2015

Accepted 15 April 2016

Edited by G. Renaud, CEA-Grenoble DSM/ INAC/SP2M/NRS, Grenoble, France

\# Present address: Evatec AG, Hauptstrasse 1a, CH-9477 Trübbach, Switzerland.

$\S$ Present address: Electron Microscopy Center, EMPA, Überlandstrasse 129, CH-8600 Dübendorf, Switzerland.

Keywords: scanning X-ray nanodiffraction; lattice bending; Ge microcrystals; thermal strain relaxation.

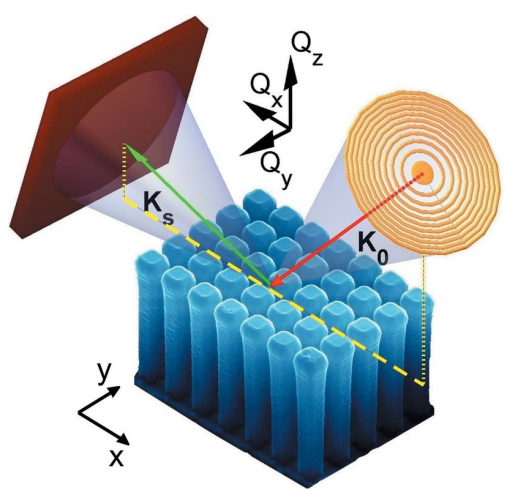

C 2016 International Union of Crystallography

\section{Lattice bending in three-dimensional Ge microcrystals studied by X-ray nanodiffraction and modelling}

\author{
Mojmír Meduňa, ${ }^{\mathrm{a}, \mathrm{b} *}$ Claudiu Valentin Falub, ${ }^{\mathrm{c}}$ Fabio Isa, ${ }^{\mathrm{c}, \mathrm{d}} \S$ Anna Marzegalli, \\ Daniel Chrastina, ${ }^{d}$ Giovanni Isella, ${ }^{d}$ Leo Miglio, ${ }^{e}$ Alex Dommann ${ }^{\text {f,g }}$ and Hans von \\ Känel ${ }^{\mathrm{c}} \S$
}

\begin{abstract}
${ }^{\mathbf{a}}$ Department of Condensed Matter Physics, Masaryk University, Kotlářská 2, CZ-61137 Brno, Czech Republic, ${ }^{\mathbf{b}} \mathrm{CEITEC}$, Masaryk University, Kamenice 5, CZ-60177 Brno, Czech Republic, 'Laboratory for Solid State Physics, ETH Zürich, OttoStern Weg 1, CH-8093 Zürich, Switzerland, 'Department of Physics, L-NESS, Politecnico di Milano, Via Anzani 42, I-22100 Como, Italy, ${ }^{\mathbf{e}}$ Department of Materials Science, L-NESS, Universitá di Milano-Bicocca, via Cozzi 55, I-20126 Milano, Italy, 'Centre Suisse d'Electronique et Microtechnique, Jaquet-Droz 1, CH-2002 Neuchâtel, Switzerland, and ${ }^{\mathbf{g}}$ Materials Meet Life, EMPA, Lerchenfeldstrasse 5, CH-9014 St Gallen, Switzerland. *Correspondence e-mail: mjme@physics.muni.cz
\end{abstract}

Extending the functionality of ubiquitous Si-based microelectronic devices often requires combining materials with different lattice parameters and thermal expansion coefficients. In this paper, scanning X-ray nanodiffraction is used to map the lattice bending produced by thermal strain relaxation in heteroepitaxial Ge microcrystals of various heights grown on high aspect ratio Si pillars. The local crystal lattice tilt and curvature are obtained from experimental threedimensional reciprocal space maps and compared with diffraction patterns simulated by means of the finite element method. The simulations are in good agreement with the experimental data for various positions of the focused X-ray beam inside a Ge microcrystal. Both experiment and simulations reveal that the crystal lattice bending induced by thermal strain relaxation vanishes with increasing Ge crystal height.

\section{Introduction}

Heterogeneous integration of high-performance materials with conventional substrates exhibiting different lattice parameters and thermal expansion coefficients has been an area of intense scientific and technological interest in the past few decades. In recent years, the topic has received special attention because of increasing demand to extend Moore's law (Moore, 1965) towards functional scaling by integrating highspeed electronic and optoelectronic devices with low-cost high-density silicon circuits. This trend, known as the 'more than Moore's law' development (Kent \& Prasad, 2008), focuses on system integration rather than increased transistor density.

However, in order to integrate of dissimilar materials one has to overcome great obstacles, such as high defect density or even cracks induced in epilayers by the large mismatch of lattice parameters and thermal expansion coefficients (Matthews et al., 1970; Fitzgerald et al., 1991). Basically, when exceeding a certain critical epilayer thickness, the mechanical stress relaxes plastically by means of misfit dislocations that are usually accompanied by threading arms extending to the surface of the epitaxial film. Moreover, the difference in thermal expansion coefficients between the substrate and the epilayer produces a thermal stress during cooling from the growth temperature which may cause wafer bending, ulti- 
mately leading to layer cracking. Dislocations and layer cracks during heteroepitaxial growth are serious problems that may render these materials of little use for any application (von Känel, 2012).

A solution to circumvent these obstacles has recently been found (Falub et al., 2012). It involves the fast epitaxial growth of the mismatched material onto an Si substrate patterned at the micrometre scale into a regular array of high aspect ratio pillars, resulting in a dense network of micrometre-sized threedimensional epitaxial crystals. The approach was initially demonstrated for the growth of $\mathrm{Ge}$ on $\mathrm{Si}(001)$ (Falub et al., 2012), and afterwards it was extended to other material combinations and crystallographic orientations (Bergamaschini et al., 2013; Falub et al., 2014; Taboada et al., 2014, 2016; Isa, Chèze et al., 2015; Isa et al., 2016). The dislocations formed in these epitaxial structures are confined near the interface with the substrate, whereas the bulk of the crystal remains defect free (Marzegalli et al., 2013; Falub et al., 2013; Isa, Pezzoli et al., 2015; Rozbořil et al., 2016).

In recent decades, X-ray diffraction has been established as an outstanding technique for determining crystal quality and revealing defects inside materials. High-resolution X-ray diffraction allows detailed nondestructive information about strain, chemical composition and defects deep inside the materials to be obtained, while averaging this information over large probed areas (Holý et al., 1999). Thus, X-ray scattering can typically be used for characterizing different types of defect in bulk materials, such as dislocations and point defects (Kaganer \& Sabelfeld, 2011; Caha et al., 2011), for determining the chemical composition in arrays of quantum nanostructures (Schmidt et al., 2002; Novák et al., 2005; Malachias et al., 2007), or for studying inter-diffusion processes in heterostructures (Meduňa et al., 2009, 2005; Ozguven \& McIntyre, 2008).

However, in some cases small crystal features at the submicrometre scale also need to be investigated, and scanning diffraction techniques using focused beams of synchrotron radiation have been addressing this requirement extensively in the past few years (Stangl et al., 2014). The development of focusing optics for X-ray beams has, for instance, permitted the measurement of the strain in thin films (Murray et al., 2005), the shape of individual quantum dots (Hanke et al., 2008), the strain inside individual quantum dots (Diaz et al., 2009; Mocuta et al., 2008) or inside nanorods (Biermanns et al., 2013), the mosaicity of graded layers (Bartosik et al., 2013; Stefenelli et al., 2013), and individual electronic devices (Hrauda et al., 2011; Paci et al., 2013), all at the nanoscale. Moreover, scanning diffraction with a focused X-ray beam has been shown to be an appropriate technique to map the lattice strain and individual defects in heteroepitaxial microstructures (Meduňa et al., 2014). Very recently, the implementation of fast scanning nanodiffraction with continuous motion was introduced by Etzelstorfer et al. (2014) and Chahine et al. (2014).

In this paper, we present a detailed theoretical modelling of $\mathrm{X}$-ray nanodiffraction on arrays of densely spaced Ge microcrystals grown epitaxially on patterned $\mathrm{Si}$ substrates and compare the models with experimental results. The work is a major extension of our previous presentation (Falub et al., 2013), in which a nanofocused X-ray beam was used to map the three-dimensional crystalline structure of individual $\mathrm{Ge}$ crystals. We demonstrate very good agreement between the experiments and the modelled data. Realistic crystal and beam shapes responsible for lowering the measurement resolution were assumed in the simulations. As mentioned already by Falub et al. (2013), the out-of-plane scattered signal could also be recorded by employing a two-dimensional detector. Thus, three-dimensional reciprocal space maps (RSMs) were reconstructed for each location at which the nanofocused $\mathrm{X}$-ray beam illuminated the crystal surface. The nanodiffraction intensity was simulated in three dimensions in this way as well. Finite element method (FEM) calculations were used to simulate the theoretical three-dimensional RSMs around the selected diffraction points and to reconstruct the theoretical position-sensitive intensity maps during the scanning nanodiffraction experiments. The local crystal lattice tilt angles in all in-plane directions, as well as the lattice curvature and strain obtained from FEM, were compared with experimental data.

\section{Samples}

The samples investigated in this work were grown by lowenergy plasma-enhanced chemical vapour deposition (Rosenblad et al., 1998). They consist of dense arrays of threedimensional Ge crystals grown epitaxially on Si wafers, which were patterned by standard optical lithography and dry reactive-ion etching into regular arrangements of micrometresized high aspect ratio pillars. The $8 \mu \mathrm{m}$ tall and $2 \times 2 \mu \mathrm{m}$ wide Si pillars were separated by $1 \mu \mathrm{m}$ wide trenches. The lithographic pattern was aligned along the crystallographic $\langle 110\rangle$ direction with a precision of around $1^{\circ}$. More information on substrate preparation and cleaning was reported by Bergamaschini et al. (2013). The epitaxial growth of the threedimensional Ge crystals was performed at a substrate temperature of $763 \mathrm{~K}$ and a deposition rate of $4.3 \mathrm{~nm} \mathrm{~s}^{-1}$, using germane $\left(\mathrm{GeH}_{4}\right)$ as the reactive gas. Coalescence of the Ge crystals is avoided in suitable growth conditions, since

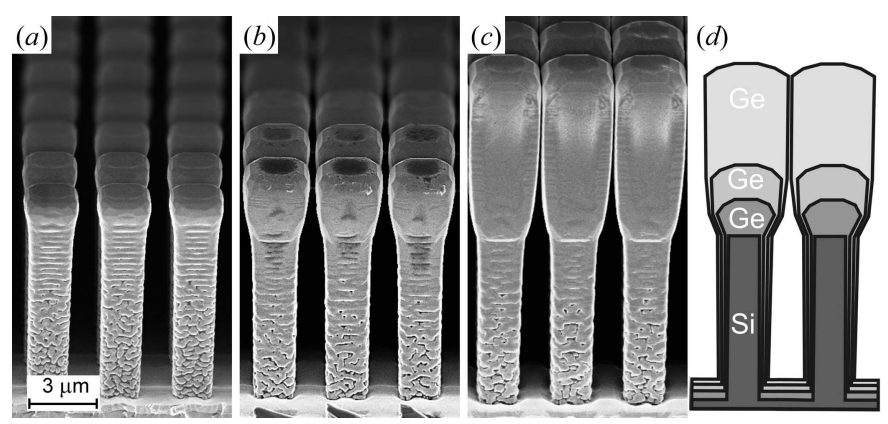

Figure 1

Perspective-view SEM micrographs of Ge crystals with heights of $(a)$ $1.2 \mu \mathrm{m},(b) 3.1 \mu \mathrm{m}$ and (c) $7.8 \mu \mathrm{m}$ grown epitaxially on $8 \mu \mathrm{m}$ tall Si pillars. (d) Schematic cross sections through two adjacent Ge/Si heterostructures, showing the approximate morphology evolution during crystal growth. 
lateral growth is self-limited by flux shielding of neighbouring crystals (Falub et al., 2012, 2014; Bergamaschini et al., 2013), which keeps them separated at a spacing of only a few tens of nanometres.

For our study, in order to follow the evolution of the crystal quality during growth, we considered Ge crystals with heights of $1.2,3.1$ and $7.8 \mu \mathrm{m}$, as shown in the scanning electron microscopy (SEM) images in Figs. 1(a)-1(c). A schematic sketch of the crystal-growth evolution depicting the progressive mechanism of self-limited lateral expansion is shown in Fig. $1(d)$.

\section{Scanning nanodiffraction experiments}

\subsection{Scattering geometry}

The large mismatch in the lattice parameters $(4.2 \%)$ and thermal expansion coefficients (e.g. $120 \%$ at room temperature) of an $\mathrm{Si}$ substrate and a planar $\mathrm{Ge}$ layer generate mechanical stresses that relax plastically by forming dislocations. The strain status of the Ge epilayer can be obtained from X-ray diffraction by mapping the reciprocal space around specific reciprocal-lattice points (RLPs) with a standard laboratory setup. However, in order to probe the strain inside an individual Ge microcrystal, the use of focused X-ray beams is mandatory.

Hence, we performed X-ray diffraction experiments on the ID01 beamline of the European Synchrotron Radiation Facility (ESRF) in Grenoble, France, which is equipped with Fresnel zone plates (FZP) (Stangl et al., 2014) so that the $\mathrm{X}$-ray beam spot size can be reduced down to $300 \times 500 \mathrm{~nm}$ (vertical $\times$ horizontal). The horizontal and vertical slits were opened such that the full $0.3 \mathrm{~mm}$ wide FZP was irradiated. The energy of the X-ray photons was $11.07 \mathrm{keV}$. Thus, the nanofocused X-ray beam, together with the high-precision $x-y$ translational piezo-stage, allows scanning of individual microcrystals (Falub et al., 2013; Meduňa et al., 2014). This technique is called scanning X-ray diffraction microscopy (SXDM) (Stangl et al., 2009; Chahine et al., 2014). The scattered intensity is then recorded at a set of mesh points along the plane parallel to the sample surface. A sketch of the experimental setup on the ID01 beamline is shown in Fig. 2(a). The sample stage was placed on a Huber goniometer which allowed scanning of the detector angular positions.

The scattered X-ray signal was measured by a two-dimensional pixel detector (MAXIPIX), and the three-dimensional RSMs were reconstructed at each $(x, y)$ point of a rectangular surface mesh. Thus, intensity frames were recorded around the symmetric 004 and asymmetric 115 reflections, and threedimensional RSMs were then built from sets of rocking scans by varying the incidence angle of the focused primary beam. An example of such a three-dimensional RSM recorded with the beam placed in between two adjacent Ge crystals is depicted in Fig. 2(b) for the $\mathrm{Si}$ and $\mathrm{Ge}(115)$ reflections. The intensity integrated along $Q_{z}, Q_{x}$ and $Q_{y}$, projected onto the $Q_{x} Q_{y}, Q_{y} Q_{z}$ and $Q_{x} Q_{z}$ planes, respectively, is plotted in the same panel. The time to collect $20 \times 20$ points of an individual surface mesh $4 \times 4 \mu \mathrm{m}$ in size at a given incidence angle was about $13 \mathrm{~min}$. We may therefore assume that the drift close to a certain diffraction point is negligible. We did, however, take into account the drift between the measurements of different reflections (004 and 115).

However, this experimental diffraction setup has several geometric limitations. The direct-space resolution is limited by the X-ray beam size and incidence angle, since along the $x$ direction (i.e. parallel to the scattering plane) the beam projection is elongated, whereas along the $y$ direction (i.e. perpendicular to the scattering plane) it is not. Thus, depending on the probed RLP, which defines the incidence angle of the primary X-ray beam, the projected beam spot size on the sample along the $x$ direction can vary. Moreover, depending on the amount of Ge material deposited on the $\mathrm{Si}$ pillars and the trench width, the inclined X-ray beam can penetrate several Ge crystals, which then all contribute to the diffracted intensity collected by the two-dimensional detector. The effect of beam penetration has also been discussed by Diaz et al. (2009) and Etzelstorfer et al. (2014). As a result, the trenches between the Ge microcrystals along the $y$ direction, perpendicular to the scattering plane, are much less resolved than those along the $x$ direction, parallel to the scattering plane.

One way to improve the resolution along the $x$ direction is to perform the scanning diffraction experiments around RLPs for which the primary beam illuminates the sample under a steep angle, such as the asymmetric reflection 115 for which the incidence angle is $\sim 47^{\circ}$. In this way, the $\mathrm{X}$-ray beam spot size is only $410 \mathrm{~nm}$ along the $x$ direction, whereas for the symmetric 004 reflection it would be $740 \mathrm{~nm}$. Further improvements to the resolution could in principle be obtained by selecting symmetric or asymmetric reflections with even steeper incidence angles. However, since the FZP and the
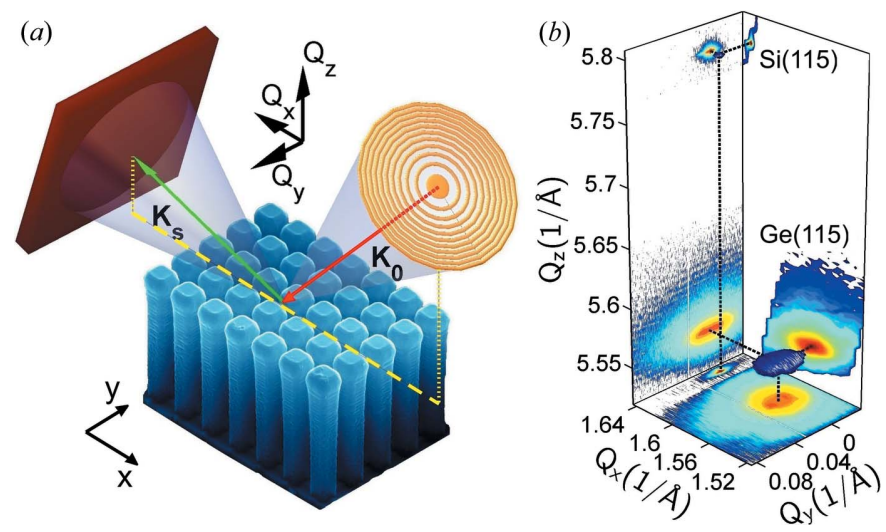

Figure 2

(a) Schematic of the experimental setup of the ID01 beamline at the ESRF, showing the scattering geometry. The primary beam $\mathbf{K}_{0}$ is focused by means of a Fresnel zone plate on the sample consisting of a dense array of epitaxial Ge crystals deposited on Si pillars; the sample is moved along the $x$ and $y$ directions by means of a piezo-stage. The scattered beam $\mathbf{K}_{\mathrm{s}}=$ $\mathbf{K}_{0}+\mathbf{Q}$ is recorded by a two-dimensional MAXIPIX X-ray detector. (b) An example of a three-dimensional RSM around the Si(115) and Ge(115) reciprocal-lattice points recorded in between two neighbouring $1.2 \mu \mathrm{m}$ tall Ge crystals. The projections on the $Q_{x} Q_{y}, Q_{y} Q_{z}$ and $Q_{x} Q_{z}$ planes show the intensity integrated along $Q_{z}, Q_{x}$ and $Q_{y}$, respectively. 
optical microscope used for locating the appropriate scanning area were positioned quite close to the sample, no reflections of higher order were accessible. Therefore, for our analysis we focused on scanning diffraction experiments performed around the asymmetric 115 reflection.

\subsection{Probing the crystal lattice bending}

The goal of our experiment is to perform three-dimensional mapping of the lattice strain and bending induced by dislocations at the Si-Ge interface and thermal stress, in individual Ge crystals.

The strain of heteroepitaxial layers is typically determined from RSMs (Holý et al., 1999), where symmetric diffraction is used to obtain the lattice tilt and normal strain, and asymmetric diffraction after tilt correction provides information on the lateral strain. Since the diffracted intensity depends on the factor $\exp (i \mathbf{h} \cdot \mathbf{u})$, where $\mathbf{h}$ and $\mathbf{u}$ are the diffraction vector and displacement field, respectively, the diffraction peak position is not sensitive to strain when $\mathbf{h} \cdot \mathbf{u}=0$. For the in-plane strain this condition is valid for measurements performed in symmetric diffraction conditions, where the displacement field is perpendicular to the diffraction vector.

Consequently, the peak position corresponds to a lattice tilt, and this can be used to correct the angular positions of other diffraction peaks. However, such tilt corrections can only be applied when the diffracted signals for all used reflections originate from exactly the same spot. Yet, in view of the great difficulty in aligning the centre of rotation at the micrometre level, this is extremely challenging, if not impossible, to realize in practice. Basically, although the piezo-stage allows accurate movement in the $x, y$ and $z$ directions, the alignment of the sample position $z$ into the centre of rotation with a precision below $\sim 100 \mathrm{~nm}$ is very complicated in view of the X-ray beam divergence of $\sim 0.08^{\circ}$. Mechanical limitations of the goniometer, such as vibrations and drift, are additional impediments. Thus, classical sample alignment by rocking curves at grazing incidence angle fails, and the sample position must be aligned independently for each scattering geometry (e.g. symmetric or asymmetric) by scanning the sample surface and matching the SXDM images for the incidence angles corresponding to various RLPs (Hrauda et al., 2011; Stangl et al., 2014).

This procedure can be used for random or irregular structures on the sample surface, which are relatively easy to identify, but it is impractical for identical periodic structures produced by patterning, such as our Ge pillars grown on prepatterned substrates. For this reason, we have performed a detailed analysis of the strain status from only the threedimensional RSMs measured around a single asymmetric reflection, e.g. 115.

The strain at a certain location can be determined from the position of the diffraction peak. However, the obtained strain information is always averaged over the volume irradiated by the X-ray beam. Despite the use of an X-ray beam focused to a submicrometre spot size, the irradiated volume is still not negligible at the scale of an individual Ge microcrystal. We assume it to be an inclined elongated cylinder with an elliptical base, penetrating the Ge crystal along the direction parallel to the incident wavevector $\mathbf{K}_{0}$. By recording three-dimensional RSMs around the asymmetric $\mathrm{Si}(115)$ and $\mathrm{Ge}(115)$ reflections for a two-dimensional $(x, y)$ surface mesh, we obtained the peak positions $\left(Q_{x} Q_{y} Q_{z}\right)$ at various locations across the $\mathrm{Ge}$ crystal array. Since along the $y$ direction the diffraction peak is not influenced by the strain and $\mathbf{h} \cdot \mathbf{u}=0$ holds, these peak positions allowed us to determine the Ge lattice tilt $\alpha_{y}=$ $\arctan \left(Q_{y} / Q_{z}\right)$ for the two-dimensional $(x, y)$ surface mesh without any further correction. From the previously discussed instrumental resolution, it also follows that it is preferable to determine the tilt along the $y$ direction, i.e. perpendicular to the scattering plane. On the other hand, we can assume the lattice bending along the $x$ and $y$ directions to be similar, in view of the fourfold symmetry of the Ge microcrystals.

Three-dimensional RSMs around the Si(115) and Ge(115) RLPs, similar to the one shown in Fig. 2(b), were constructed for each point in a two-dimensional $(x, y)$ rectangular mesh, $\sim 4 \times 4 \mu \mathrm{m}$ in size, covering four adjacent Ge crystals. The total intensity at a certain $(x, y)$ location was then determined by integrating the signal around the three-dimensional Ge peak. Position-sensitive maps, discussed also by Falub et al. (2013), showing the total intensity collected around the $\mathrm{Ge}(115)$ peak while scanning the X-ray beam across the $(x, y)$ mesh are shown in Fig. 3 for all three Ge crystal heights. It is evident from these maps that, when the Ge crystal height increases, the diffracted signal becomes smeared out along the $x$ direction, i.e. parallel to the scattering plane, owing to beam penetration through several neighbouring Ge crystals. For the tallest Ge crystals $(7.8 \mu \mathrm{m})$, the separation between adjacent Ge microcrystals is practically undetectable, since in this case they are separated by only $\sim 30 \mathrm{~nm}$. Thus, while the trenches parallel to the $x$ direction are visible, those parallel to the $y$ direction have completely vanished.

In fact, the $(x, y)$ maps in Fig. 3 provide the location of the $\mathrm{X}$-ray beam on the Ge crystal array. A few particular scanning positions are indicated by white symbols, i.e. in the middle of a Ge crystal, between two adjacent crystals in the vertical and horizontal trenches, and at the crossing of two trenches. For the tall Ge crystals an even larger area was measured, but we could not find better resolved separations between the crys-

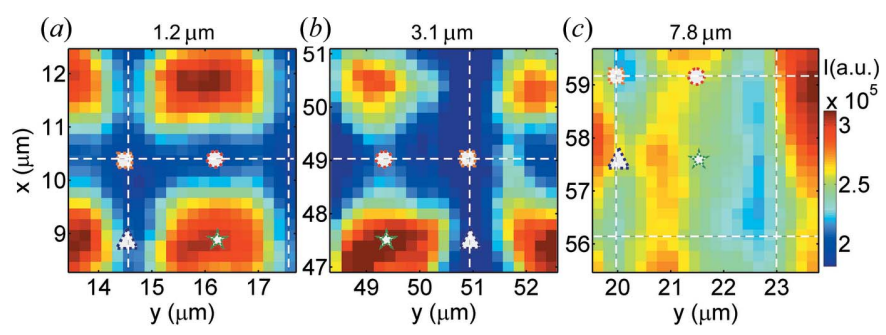

Figure 3

Position-sensitive maps of the total scattered intensity around the $\mathrm{Ge}(115)$ reflection recorded over four Ge crystals with various heights: (a) $1.2 \mu \mathrm{m}$, (b) $3.1 \mu \mathrm{m}$ and (c) $7.8 \mu \mathrm{m}$. The white symbols indicate particular scanning locations of the focused X-ray beam, i.e. the middle of a Ge crystal, in between two adjacent crystals along the horizontal and vertical trenches, and the intersection between two trenches. 
tals. The areas in Figs. 3(a)-3(c) were selected to be of the same size for comparison. As a guide for the crystal borders we can also use the crystal tilt map of Fig. 5(c) below.

For the measured samples it was found that, as the X-ray beam moves across the $(x, y)$ mesh of Fig. 3, the most evident changes in the peak shape in reciprocal space are revealed in the $Q_{x} Q_{y}$ plane, parallel to the $x y$ plane in real space. Some of these RSMs are shown in Fig. 4; the data for the $1.2 \mu \mathrm{m}$ tall crystals were previously demonstrated by Falub et al. (2013). By scanning the beam along $x$ across several crystals, we found the position of the Ge diffraction peak in the $Q_{x}$ direction to vary periodically, while the position in the $Q_{y}$ direction changes periodically along the $y$ direction. On the other hand, we did not observe any change in the $Q_{z}$ position while the $\mathrm{X}$-ray beam was scanning the $(x, y)$ surface mesh. Moreover, we observed that, when the X-ray beam is located in a trench between two Ge crystals, the diffraction peak exhibits two maxima oriented along the $Q_{x}$ or $Q_{y}$ direction, depending on whether the trench is perpendicular or parallel to the scattering plane (see Fig. 4). When the beam is located at the intersection of two trenches, the diffraction peak even exhibits four maxima, whereas when it hits the middle of a crystal only one peak is observed.

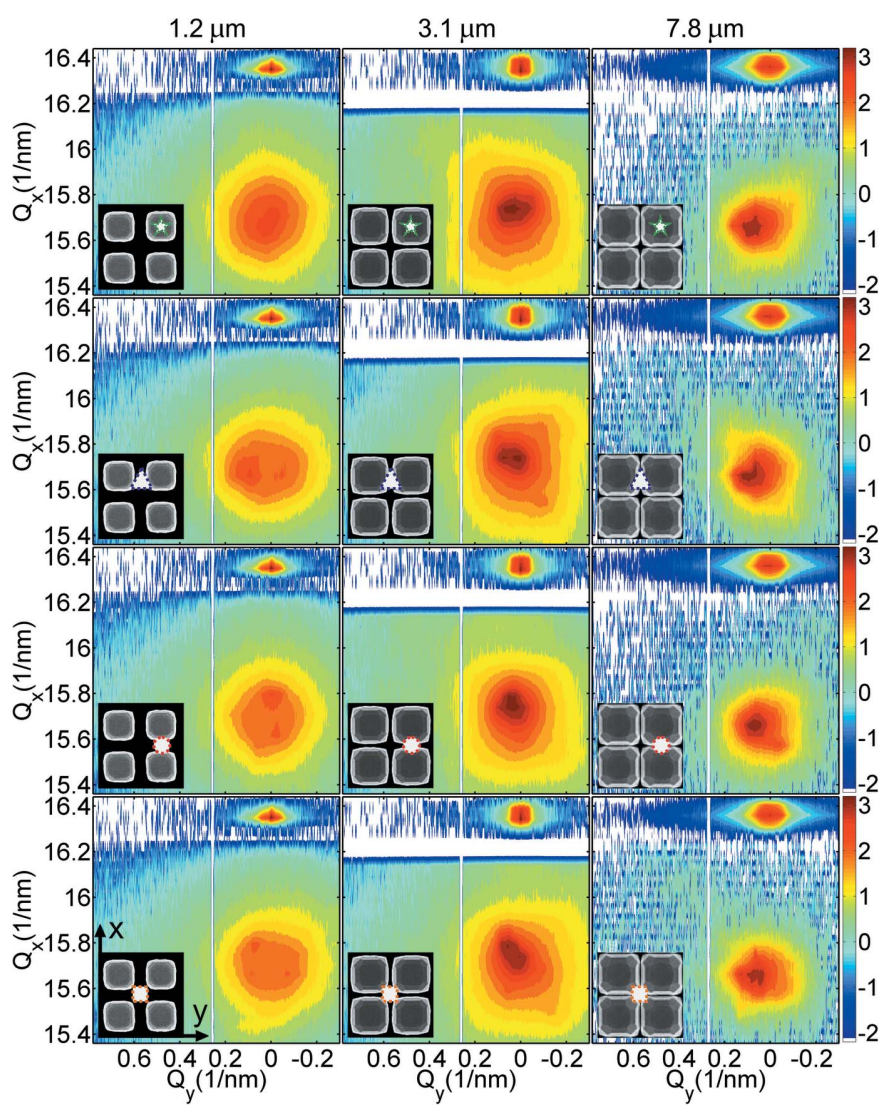

Figure 4

$Q_{x} Q_{y}$ projections of the three-dimensional RSMs measured around the $\mathrm{Ge}(115)$ reflection for various crystal heights: $1.2 \mu \mathrm{m}$ (left column), $3.1 \mu \mathrm{m}$ (middle column) and $7.8 \mu \mathrm{m}$ (right column). Each row corresponds to the particular scanning position depicted by the white symbols in the insets in the lower left-hand corners. The insets show a square area measuring $6 \times 6 \mu \mathrm{m}$ of four probed crystals.
These variations in peak maxima are most pronounced for shorter Ge crystals and diminish as the crystals become taller. Thus, the splitting of the diffraction peak is clearly visible for the $1.2 \mu \mathrm{m}$ tall crystals, barely detectable for the $3.1 \mu \mathrm{m}$ tall crystals and entirely absent for the $7.8 \mu \mathrm{m}$ tall crystals. The continuous change in the peak position during the scanning diffraction experiment is a result of the variable crystal lattice tilt caused by the thermal strain. We used the peak positions $Q_{y}$ at every $(x, y)$ point to calculate the local lattice tilt $\alpha_{y}$. This tilt varies linearly as a function of position across each individual Ge crystal with a slope depending on the crystal height. The $\left(Q_{x} Q_{y} Q_{z}\right)$ positions of the peaks in the data set were defined as the centre of mass of data points lying in an area around the total maximum, where the area size coincides with the peak width. Whenever more than one local maximum was observed in the three-dimensional RSM, the centre of mass was calculated independently for each local maximum. On a larger scale, $\alpha_{y}$ thus varies in a saw-tooth manner with the periodicity of the substrate pattern. The saw-toothed behaviour of the lattice tilt is quite evident for the $1.2 \mu \mathrm{m}$ tall crystals, but for the 3.1 and $7.8 \mu \mathrm{m}$ tall crystals it is at the detection limit (see Fig. 5).

We tentatively assign the random deviations of $\alpha_{y}$ from linearity to variations in the lattice parameter due to crystal defects close to the $\mathrm{Ge}-\mathrm{Si}$ interface, such as misfit dislocations (and their associate strain fields), or to random noise.

The linear dependence of the lattice tilt $\alpha_{y}$ along the $y$ direction within a Ge crystal is equivalent to the bending of the crystal lattice, with the corresponding radius of curvature given by the reciprocal value of the first derivative of the tilt at position $y: R=\left(\Delta Q_{y} / Q_{z} / \Delta y\right)^{-1}$. Thus, for the $1.2 \mu \mathrm{m}$ tall crystals the average radius of curvature of the bent lattice planes is $R=770 \pm 20 \mu \mathrm{m}$. For the $3.1 \mu \mathrm{m}$ tall crystals the lattice bending is much smaller, $R=10 \pm 8 \mathrm{~mm}$, close to the detection limit of our measurement. Finally, for the $7.8 \mu \mathrm{m}$ tall crystals we could not observe any sign of lattice bending, so we consider the radius of curvature to be infinite.

The strain at a specific location can only be assessed correctly if one knows the lattice tilt at that site. As we have

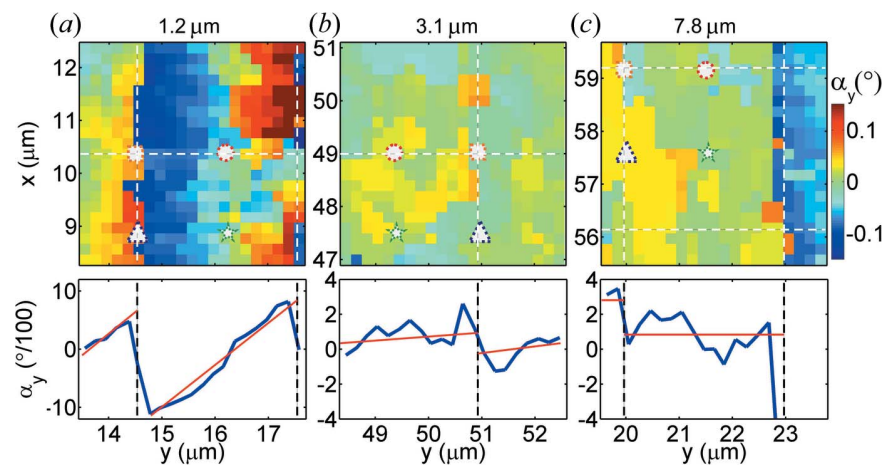

Figure 5

Position-sensitive maps of the lattice tilt $\alpha_{y}$, obtained from the Ge(115) peak positions along the $Q_{y}$ direction for various Ge crystal heights: $(a)$ $1.2 \mu \mathrm{m},(b) 3.1 \mu \mathrm{m}$ and $(c) 7.8 \mu \mathrm{m}$. The scanned $(x, y)$ area in the top panels is exactly the same as in Fig. 3. The lattice tilt dependence in the bottom panels was obtained by averaging the top panels over the $x$ position. 
mentioned earlier, a reliable estimation of the lattice tilt is only possible along the $Q_{y}$ direction, i.e. perpendicular to the scattering plane. Moreover, in order to determine the lattice parameters, the $Q_{x}$ and $Q_{z}$ positions of the diffraction peak have to be corrected by the local lattice tilt in the $Q_{x} Q_{z}$ plane. However, in view of the fourfold symmetry of the Ge crystals one can assume equal lattice bending along the $Q_{x}$ and $Q_{y}$ directions. The lattice bending inside the Ge crystals can then be approximated by a concave circular paraboloid, with the radius of curvature at the minimum equal to the average value of $R$ calculated previously. Yet, it should be noted that this approximation neglects any strain variation caused by local defects, i.e. it assumes an ideal bending of the crystal lattice. Still, it can be used for correcting the $Q_{x}$ and $Q_{z}$ positions of the 115 RLP.

After the lattice bending correction, one can determine the lattice parameters $a_{x}$ and $a_{z}$, the in-plane $\varepsilon_{\|}=\varepsilon_{x x}=\varepsilon_{y y}$ and the normal $\varepsilon_{\perp}=\varepsilon_{z z}$ strain components, the Ge content, and the relaxation degree over the $(x, y)$ surface mesh following standard procedures (Holý et al., 1999). Thus, we found that the lattice parameters $a_{x}$ and $a_{z}$ exhibit no significant variation over the two-dimensional surface mesh scanned by the beam, the perceived variations being due to measurement noise whose amplitude decreases with increasing crystal height. The degree of relaxation for all crystal heights, i.e. 1.2, 3.1 and $7.8 \mu \mathrm{m}$, was found to be $1.00 \pm 0.08$, which demonstrates that the Ge crystals grown on Si pillars are completely relaxed. Moreover, the Ge content across the $(x, y)$ surface mesh was $0.98 \pm 0.02$ for all samples. Finally, the in-plane strain $\left|\varepsilon_{\|}\right|$for the $1.2,3.1$ and $7.8 \mu \mathrm{m}$ tall Ge crystals for all positions in the measured area was well below $0.5,0.4$ and $0.2 \%$, respectively, whereas the normal strain $\varepsilon_{\perp}$ was below $0.3,0.2$ and $0.1 \%$, respectively. According to the FEM calculations presented in the next section, we expect that both the in-plane and the normal strains are in fact much smaller, but owing to measurement noise we were not able to map strain variation reliably in microcrystals, except the lattice tilt.

As we have seen, Ge crystal lattice bending approximated by a circular paraboloid can easily be estimated from the periodic shift of the diffraction peaks while the X-ray beam scans the array of microcrystals. These shifts are similar for both the $Q_{x}$ and $Q_{y}$ directions, since the microcrystals are on average completely relaxed and the strain contribution to the peak positions can be neglected even for asymmetric diffraction. The presence of misfit dislocations at the Si-Ge interface is usually responsible for diffraction peak broadening (Kaganer et al., 1997) and most likely accounts for the random variations in the intensity background of the diffraction peaks observed during beam scanning.

\subsection{Three-dimensional FEM simulations of thermal strain relaxation}

In order to explain the diffraction peak variations observed in the RSMs while scanning individual Ge microcrystals with the nanofocused X-ray beam, we performed three-dimensional FEM calculations of the strain distribution inside the
Ge crystals with heights of $1.2,3.1$ and $7.8 \mu \mathrm{m}$. These calculations have as input the different thermal expansion coefficients of $\mathrm{Ge}$ and $\mathrm{Si}$, and the temperature change upon cooling the Ge crystals from the growth temperature to room temperature. The three-dimensional crystal geometries were built on the basis of top- and cross-sectional SEM images. An initial hydrostatic expansion of the Ge pillar of $0.20 \%$ was considered as derived from the difference in the thermal expansion coefficients of $\mathrm{Si}$ and $\mathrm{Ge}$ for a temperature difference of $500 \mathrm{~K}$. The different thermal expansion coefficients of Ge and Si result in strain in the form of concave lattice bowing. Similar results for the 1.2 and $3.1 \mu \mathrm{m}$ tall crystals have been obtained previously by Falub et al. (2013), although the FEM simulations presented in this work were recalculated for more realistic crystal shapes in order to obtain better agreement between the measured and simulated diffracted intensities.

The FEM simulations were performed using the Multiphysics package (COMSOL, Stockholm, Sweden) by meshing the three-dimensional geometries with 480324,353560 and 328316 elements, respectively, from the thickest to the smallest crystals. They provided all six components of the displacement gradient tensor $J_{i j}=\varepsilon_{i j}+\Omega_{i j}, i, j=x, y, z$, which, together with the boundary conditions, were used to calculate the displacement field $\mathbf{u}=\left(u_{x}, u_{y}, u_{z}\right)$ around a certain reference point. Here, $\varepsilon$ represents the symmetric strain tensor and $\Omega$ the antisymmetric rotation tensor.

The $\varepsilon_{x x}$ and $\varepsilon_{z z}$ components of the three-dimensional strain tensor are shown in Fig. 6, superimposed on the sliced crystal models for different crystal heights. To find the lattice bending, we calculate the asymmetric rotation tensor $\Omega$ whose components $\Omega_{i j}(x, y, z)$ directly represent the angles of rotation of the elementary cell. However, because of the fourfold symmetry of the crystals we calculated only the $\Omega_{y z}(x, y, z)$ components, since $\Omega_{x z}=\Omega_{y z}$. The $\Omega_{y z}$ values represent small local rotation angles of the lattice planes around the $x$ axis at the point $\mathbf{r}=(x, y, z)$ and therefore can also be written as the derivative of $\Delta z$ with respect to $y(\mathrm{~d} \Delta z / \mathrm{d} y) . \Delta z$ can be understood as the vertical deviation along $z$ of crystallographic planes from their unbent planar state when $z=$
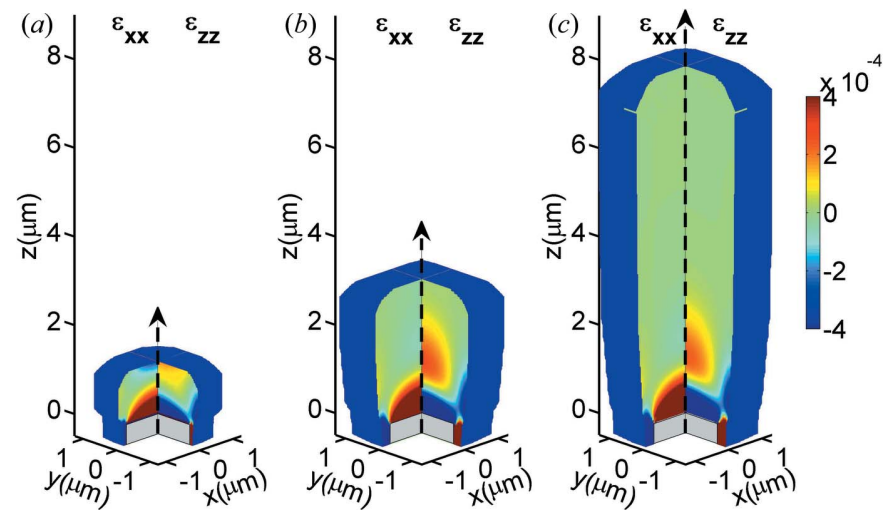

Figure 6

Three-dimensional FEM calculations of the strain tensor components $\varepsilon_{x x}$ and $\varepsilon_{z z}$ inside Ge crystals with heights of $(a) 1.2 \mu \mathrm{m},(b) 3.1 \mu \mathrm{m}$ and $(c)$ $7.8 \mu \mathrm{m}$. The dashed arrows represent the crystal axes along the growth direction. 
constant. The lattice bending in the $y z$ plane can then be calculated by integrating for any $z$ position, $\Delta z(y)=$ $\int \Omega_{y z}(x, y, z) \mathrm{d} y$. In Fig. 7 we show two-dimensional maps of the rotation tensor component $\Omega_{y z}(0, y, z)$ in the middle of $\mathrm{Ge}$ crystals having different heights, onto which we have superimposed a sketch of the bent crystal lattice whose scale has been multiplied by a factor of 200 for better visibility. Thus, one clearly sees (Fig. 8) that the lattice bending diminishes towards the top of the crystal, as expected. Moreover, the bending at the top drops rapidly with increasing crystal height.

On the other hand, the lattice bending at a given distance $z$ from the SiGe interface is quite similar for all crystals. The largest differences occur close to the $\mathrm{Si}-\mathrm{Ge}$ interface, but $\sim 1 \mu \mathrm{m}$ above the interface the strain no longer differs
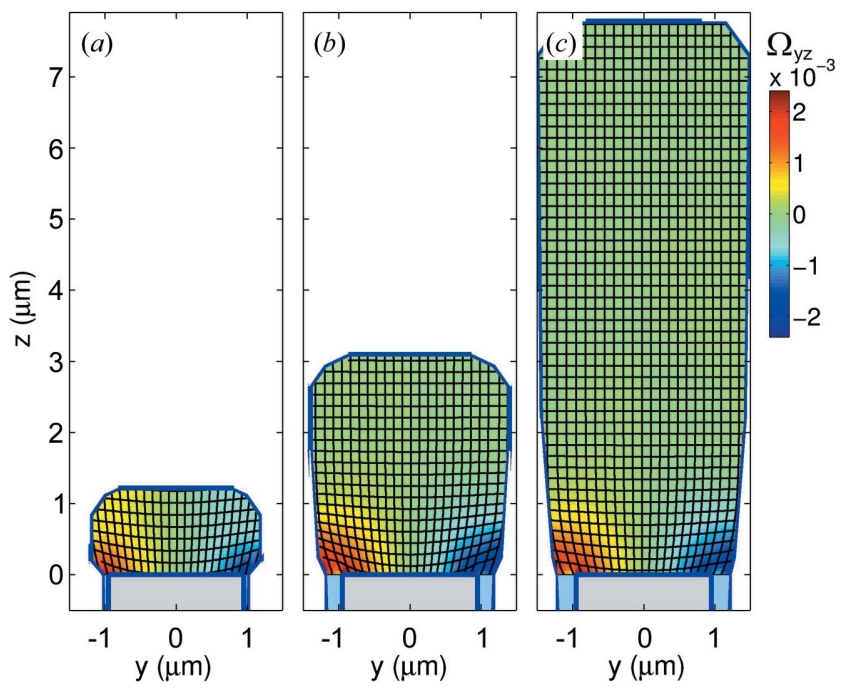

Figure 7

Lattice bending calculated from the rotation tensor for $(a) 1.2 \mu \mathrm{m},(b)$ $3.1 \mu \mathrm{m}$ and (c) $7.8 \mu \mathrm{m}$ tall Ge crystals. The colour maps represent cross sections through the $y z$ component of the three-dimensional rotation tensor sliced in the middle of the Ge crystals $(x=0)$. The scale of the lattice bending was increased by a factor of 200 to enhance the visibility of the local strain.

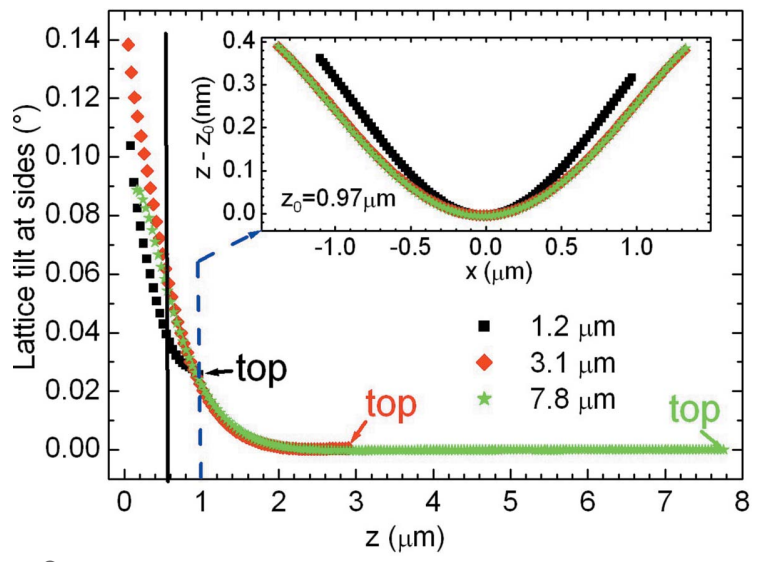

Figure 8

The tilt of the lattice planes at the crystal side walls, as obtained from the FEM calculations for the $1.2,3.1$ and $7.8 \mu \mathrm{m}$ tall crystals. The inset shows a comparison of the lattice bending at a distance $z=1 \mu \mathrm{m}$ (dashed blue line) from the $\mathrm{Si}-\mathrm{Ge}$ interface for the three different $\mathrm{Ge}$ crystals, represented by different colours. significantly for crystals with different heights. For taller crystals the strain vanishes rapidly with height, and for the 3.1 and $7.8 \mu \mathrm{m}$ tall crystals the lattice bending is practically the same at $z=1 \mu \mathrm{m}$ above the $\mathrm{Si}-\mathrm{Ge}$ interface (see inset of Fig. 8). In view of the penetration depth of the X-rays, the lattice bending obtained from the X-ray analysis is averaged over the whole Ge crystal, and hence cannot be compared directly with the calculated bending. The observed average bending does, however, correspond to the value obtained by the threedimensional simulations at a $z$ value of half the crystal height. The lattice bending estimated from the scanning X-ray nanodiffraction experiments is therefore in very good agreement with the FEM calculations.

Moreover, the strain values obtained from the nanodiffraction experiments are in very good agreement with those simulated by three-dimensional FEM. Thus, both the lateral and normal strain values from FEM are below $0.2 \%$ for the $1.2,3.1$ and $7.8 \mu \mathrm{m}$ tall Ge crystals.

\subsection{Simulations of the scanning X-ray nanodiffraction experiments}

In order to obtain a still deeper insight into the scanning nanodiffraction experiments and the evaluation of the experimental data presented in $\$ 3.2$, we simulated the scattered intensity distribution in the three-dimensional reciprocal space around the 115 RLP for defined positions of a focused theoretical X-ray beam, similar to the experimental one, by using the three-dimensional FEM crystal model discussed in the previous section. Thus, after calculating the strain tensor for realistic geometric models based on SEM micrographs such as the one in Fig. 9(a), we simulated the three-dimensional RSMs while the hypothetical X-ray beam scans the three-dimensional crystal model.

Basically, on the basis of the FEM data we first built the three-dimensional matrices of the displacement vector $\mathbf{u}(\mathbf{r})=$ $\left(u_{x}, u_{y}, u_{z}\right)$ with $256 \times 256 \times 256$ elements, corresponding to a realistic crystal model. From scattering theory, the intensity of $\mathrm{X}$-rays diffracted by a strained crystal lattice is proportional to the Fourier transform of the factor $\exp (i \mathbf{h} \cdot \mathbf{u})$, where as before $\mathbf{h}$ is the diffraction vector in reciprocal units, e.g. $h=$ $\left(2 \pi / a_{\mathrm{Ge}}\right)(1,1,5)$, and $\mathbf{u}$ is the displacement vector in units of the lattice parameter $a_{\mathrm{Ge}}$. In Fig. $9(b)$ we show the real component of the factor $\exp (i \mathbf{h} \cdot \mathbf{u})$ in the middle of the $3.1 \mu \mathrm{m}$ tall crystal. The scattered intensity at a certain location can be expressed by the differential cross section, which is proportional to the square of the Fourier transform of the electron density in the region probed by the X-ray beam, $\rho_{\mathbf{h}}(\mathbf{r})$, statistically averaged over the ensemble of all possible random structural deviations:

$$
\frac{\mathrm{d} \sigma}{\mathrm{d} \Omega} \simeq\left\langle\left|\rho_{\mathbf{h}}^{\mathrm{FT}}(\mathbf{q})\right|^{2}\right\rangle
$$

Here $\rho_{\mathbf{h}}$ is defined by following equation (Holý et al., 1999):

$$
\rho_{\mathbf{h}}(\mathbf{r}) \simeq\left\{\chi_{\mathbf{h}}(\mathbf{r}) \exp [i \mathbf{h} \cdot \mathbf{u}(\mathbf{r})]-1\right\} S_{0}(\mathbf{r}) \exp [-\operatorname{Im}(\mathbf{q}) \cdot \mathbf{r}]
$$

This expression takes into account the hth Fourier component of the crystal polarizability $\chi_{\mathbf{h}}(\mathbf{r})$ determined by the Ge 
material, the factor $\exp (i \mathbf{h} \cdot \mathbf{u})$ displayed in Fig. $9(b)$, the shape function of the X-ray beam irradiating the sample $S_{0}(\mathbf{r})$ and an absorption factor along the beam path $\exp [-\operatorname{Im}(\mathbf{q}) \cdot \mathbf{r}]$ inside the crystal. The shape function of the X-ray beam, $S_{0}(\mathbf{r})$, is defined as 1 inside the beam at maximum intensity and zero outside the beam.

For simplicity, we have taken the penetration depth as obtained from the ratio of the wavelength and the imaginary part of the polarizability $\chi_{0}$, which for the selected energy amounts to around $70 \mu \mathrm{m}$ for perpendicular incidence. The actual penetration depth depends strongly on the incidence angle with respect to the crystal facet, on whether we are close to or far from a diffraction peak, and on whether the geometry applies to the Laue or Bragg case, but it still reaches much more than $10 \mu \mathrm{m}$. Since even the tall crystals are smaller than this, we think that absorption inside the crystals can in fact be neglected in all samples. Owing to the small crystal size and steep incidence angles, we neglect dynamic diffraction and refraction effects and assume kinematic scattering only.

For our calculations, we assumed an X-ray beam with a Gaussian profile having a circular symmetry in cross section with an FWHM of $500 \mathrm{~nm}$. A sketch of the beam path through the crystal, together with its cross section, is shown in Fig. 9(a).

(a)

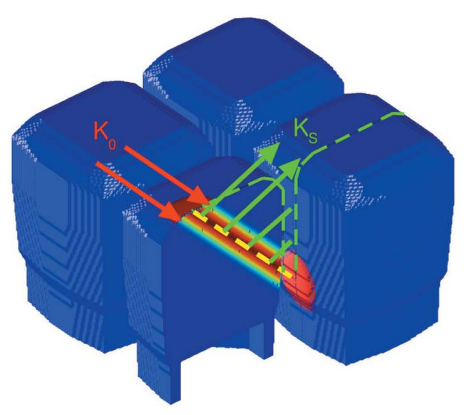

(b)

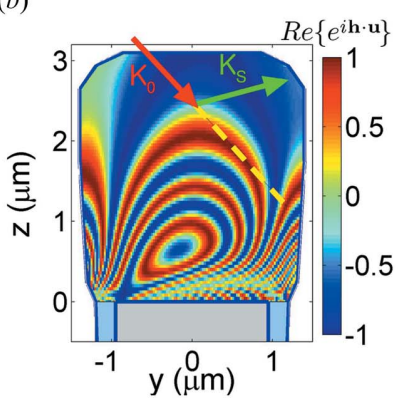

(c)

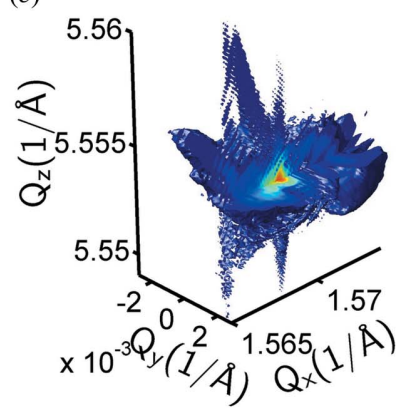

Figure 9

(a) A perspective view of a three-dimensional FEM model consisting of four adjacent $3.1 \mu \mathrm{m}$ tall Ge crystals on Si pillars, constructed on the basis of SEM micrographs. One of the crystals is irradiated by the nanofocused $\mathrm{X}$-ray beam at the location indicated by the red spot. The irradiated crystal is sliced to show the beam path. The X-ray beam enters the top of the crystal, exits on the side wall, and then irradiates an adjacent crystal. (b) The two-dimensional $(y, z)$ distribution of the real component of the function $\exp (i \mathbf{h} \cdot \mathbf{u})$ in the middle of the $3.1 \mu \mathrm{m}$ tall Ge crystal for the asymmetric 115 scattering geometry. The red arrow represents the incident beam wavevector along the beam path (yellow dashed line) and the green arrow indicates the exit wavevector. (c) The simulated scattered intensity for the scanning nanodiffraction experiment depicted in panel (a) and the factor $\exp (i \mathbf{h} \cdot \mathbf{u})$ shown in panel $(b)$, represented as a threedimensional isocontour plot.
Using equations (1) and (2) we calculated the three-dimensional RSMs around the 115 reflection for every mesh point in the three-dimensional crystal model scanned by the theoretical X-ray beam. An example of such a simulated threedimensional RSM for a beam probing the middle of a $3.1 \mu \mathrm{m}$ tall Ge crystal is shown in Fig. 9(c).

In order to include the complete beam path, the periodic arrangement of the microcrystals was taken into account in the calculations, since the beam can even penetrate three crystals, especially those which are $7.8 \mu \mathrm{m}$ tall. Because of their small width, beams from neighbouring crystals do not interfere with each other. For the calculations we have used a data set containing $2 \times 2$ crystals repeated periodically in the plane of the sample surface. Since the Ge layer on the side walls of the Si pillars is highly dislocated, as we know from TEM, we do not expect any coherent contribution to the diffraction from this area. Also from FEM, it follows that the strain is so high in this region that the material is plastically relaxed. Thus, we think that the thin layer on the side walls of the Si is mainly responsible for the broad background in the RSMs, similar to the signal from material in the trenches. These contributions were therefore not included in the calculations.

In Fig. 3 we present the position-sensitive maps of the total X-ray intensity collected around the Ge(115) RLP. These maps clearly reveal the shape of the Ge crystals, at least for the 1.2 and $3.1 \mu \mathrm{m}$ tall crystals, since the total diffracted intensity is proportional to the crystal volume probed by the X-rays.

Fig. 10 shows the corresponding simulated total intensity maps for all three investigated crystal heights. The measured (Fig. 3) and simulated position-sensitive total intensity maps for the $1.2 \mu \mathrm{m}$ crystals agree very well. However, for the $3.1 \mu \mathrm{m}$ crystals, and especially for the $7.8 \mu \mathrm{m}$ tall crystals, the agreement between experiment and simulation is not as good. For all three crystal heights, the simulations show that the crystal separations are less resolved along the $y$ direction. For the $7.8 \mu \mathrm{m}$ tall crystals we essentially see only the trenches along the $x$ direction. The experimental observation of trenches oriented along the $x$ direction is even more difficult for the $7.8 \mu \mathrm{m}$ tall crystals. The intensity variation across the $\mathrm{Ge}$ crystals decreases with increasing Ge crystal height for both
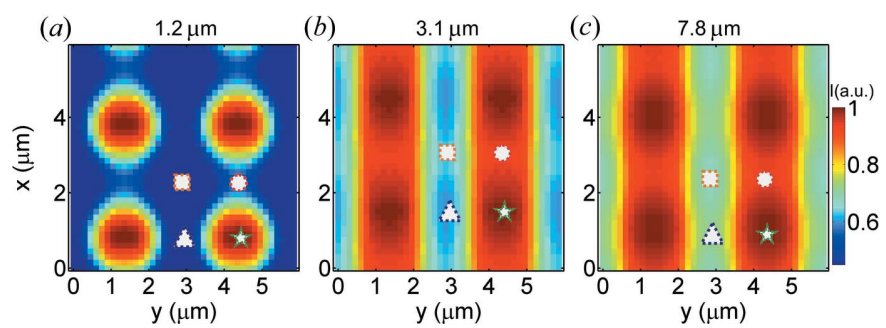

Figure 10

Simulated position-sensitive surface maps of the total intensity around the $\mathrm{Ge}(115)$ peak during beam scanning of an array of four Ge crystals having different heights: (a) $1.2 \mu \mathrm{m}$, (b) $3.1 \mu \mathrm{m}$ and (c) $7.8 \mu \mathrm{m}$, corresponding to the experimental results of Fig. 3 . The white symbols indicate particular scanning locations of the focused X-ray beam, e.g. the middle of a Ge crystal, in between two adjacent crystals along the horizontal and vertical trenches, and the intersection between a horizontal and a vertical trench, also indicated in Fig. 11. 
measurements and simulations. As already discussed in $\$ 3.2$, for the $7.8 \mu \mathrm{m}$ tall crystals the measured data do not permit us to distinguish reliably between different crystals, either in the $x$ or in the $y$ direction. The positions marked in Fig. 3(c) are a guess based on both the intensity and the 'jump' in the lattice tilt at $y=23 \mu \mathrm{m}$, by means of which different neighbouring rows of crystals are detected. We note that, in the theoretical model, we do not take into account the strain fields induced by the dislocations present at the $\mathrm{Si}-\mathrm{Ge}$ interface, and the random net tilt of the individual Ge crystals is also neglected. This explains why the calculated total intensity maps in Fig. 10 are very regular compared with the experimental ones. The random net crystal tilt observed from different measurements on individual crystals (Falub et al., 2013) or from ensembles of crystals (Rozboril et al., 2016) varies in the range $0.03-0.07^{\circ}$. From all the panels in Fig. 5 we deduce a slightly smaller value of around $0.02^{\circ}$.

Fig. 11 shows a series of $Q_{x} Q_{y}$ projections of the simulated three-dimensional RSMs corresponding to four particular locations: the middle of the crystal, between two adjacent

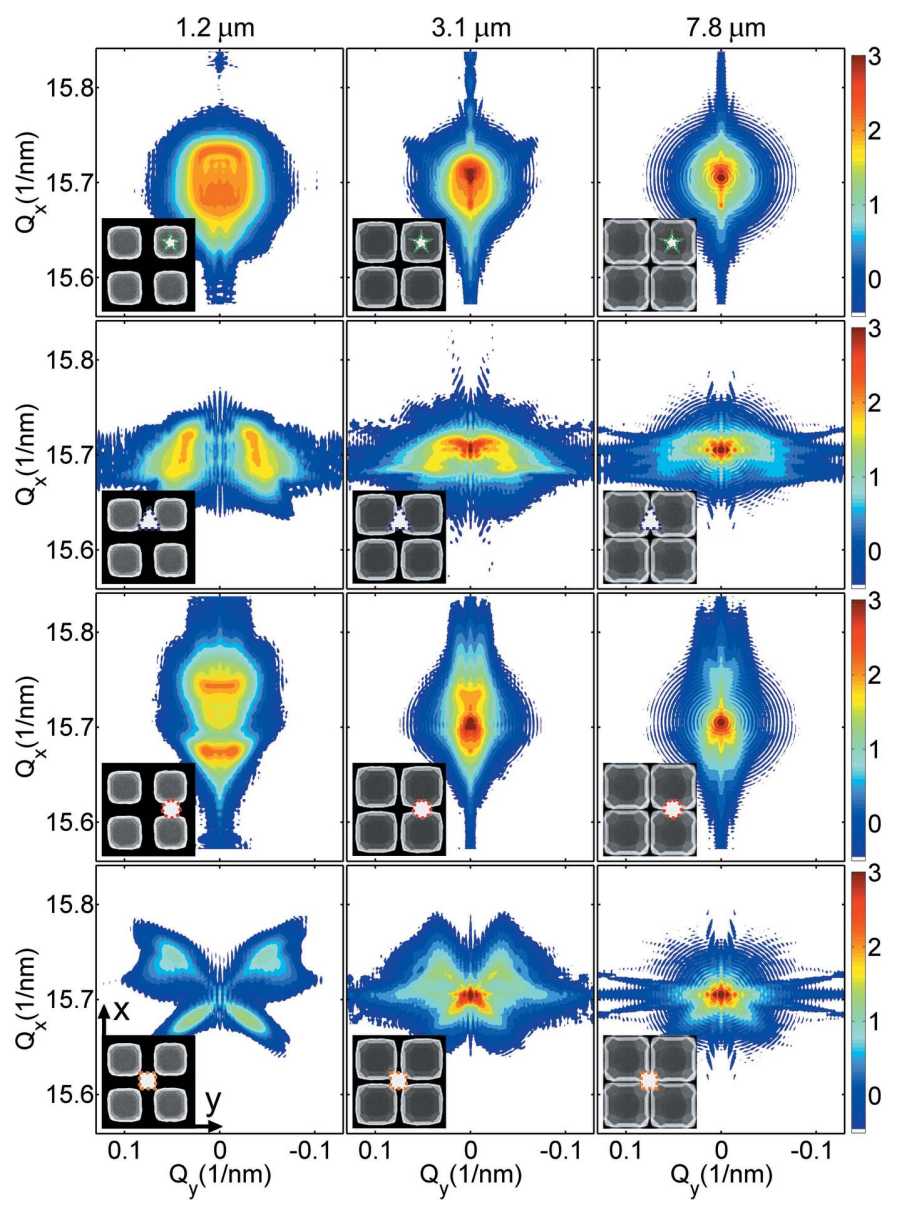

Figure 11

$Q_{x} Q_{y}$ projections of the simulated three-dimensional RSMs around the Ge(115) reflection, based on three-dimensional FEM data for four different locations of the theoretical X-ray beam with size $500 \times 400 \mathrm{~nm}$, similar to Fig. 4. The left, middle and right columns correspond to the 1.2, 3.1 and $7.8 \mu \mathrm{m}$ tall Ge crystals, respectively. The insets show a square area measuring $6 \times 6 \mu \mathrm{m}$ of four probed crystals, where the particular beam position is depicted by the white symbols. crystals in the vertical or horizontal trench, and at the trench crossing, for all three different crystal heights. Similar to the experimental data (see Fig. 4), the simulations reveal the diffraction peak splitting caused by thermal bending, depending on the orientation of the trenches. Basically, the peak splitting is caused by the simultaneous irradiation of distinct areas of two adjacent crystals with opposite lattice tilts. Consequently, the separation between the two peak components is proportional to the average lattice curvature inside the Ge crystal, and therefore it diminishes with increasing crystal height.

More insight into the lattice tilt variation within the Ge crystals can be obtained by comparing the experimental and simulated $\left(y, Q_{y}\right)$ maps of the intensity maximum of the Ge(115) peak in the $Q_{x} Q_{y}$ plane for a fixed position $x$, approximately in the middle of the crystals. This comparison is shown in Fig. 12 for the three crystal heights. It reveals the zigzag behaviour of the diffraction peak maximum along $Q_{y}$ when the X-ray beam moves along the $y$ direction. The calculated and measured data are in very good agreement. Thus, for the $1.2 \mu \mathrm{m}$ tall crystals, the intensity maximum of the diffraction peak exhibits a linear dependence on $y$ when the beam moves across one Ge crystal, while displaying the typical periodic sawtooth behaviour already observed in Fig. 5 when the beam moves across more crystals. Experimentally, this behaviour is less clear for the $3.1 \mu \mathrm{m}$ tall crystals, being obscured by measurement noise. Nevertheless, the simulations fit very well with the measurements. Finally, for the $7.8 \mu \mathrm{m}$ tall crystals the zigzag behaviour is neither observed nor theore-
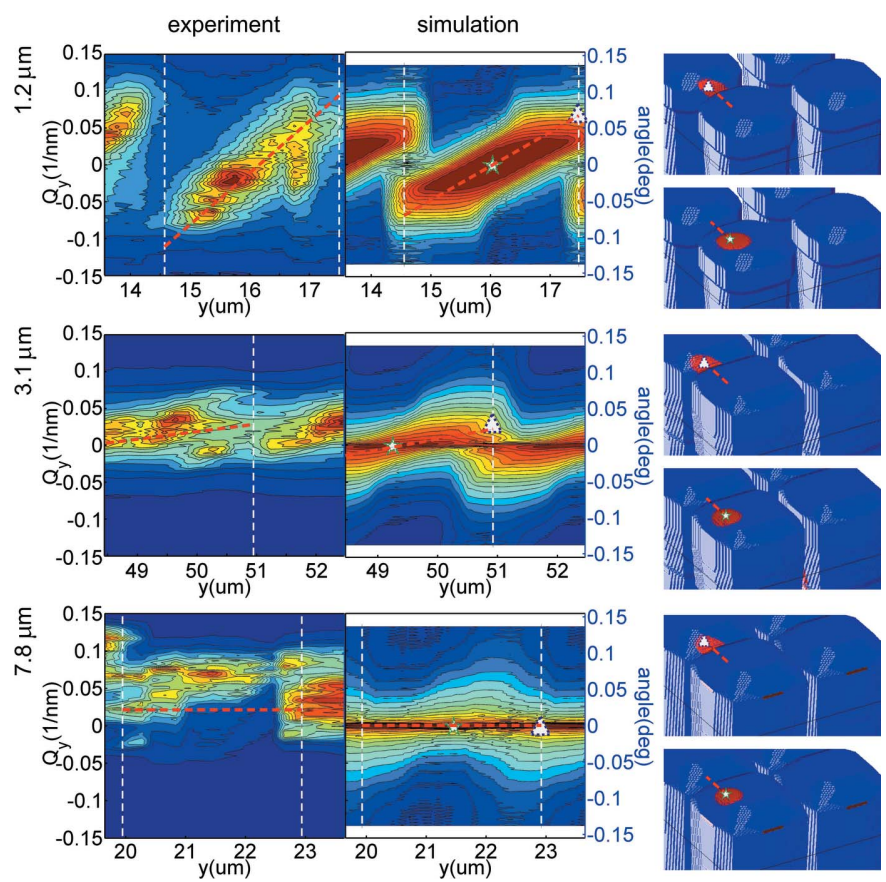

Figure 12

Experimental (left column) and simulated (middle column) $\left(y, Q_{y}\right)$ distributions for selected fixed $x$ positions corresponding to $1.2 \mu \mathrm{m}$ (top row), $3.1 \mu \mathrm{m}$ (middle row) and $7.8 \mu \mathrm{m}$ (bottom row) tall crystals. The white symbols correspond to particular positions of the nanofocused $\mathrm{X}$-ray beam shown in the right-hand column. 
tically expected. From the slope of the linear dependence one can determine the average lattice curvature, as explained in \$3.2. Thus, both the experiment and the simulations show that the average lattice curvature decreases with increasing $\mathrm{Ge}$ crystal height, and the experimental value agrees well with the simulated value. Since the X-ray simulations are based on FEM calculations, the same can be said concerning the strain, which becomes negligible towards the top of crystals taller than $3 \mu \mathrm{m}$.

Moreover, we observed that the shape of the $\left(y, Q_{y}\right)$ maps depends strongly on the shape of the beam. Thus, the best agreement between experiment and simulation was found for a Gaussian beam profile having a width between 500 and $700 \mathrm{~nm}$. This compares well with the nominal width of the $\mathrm{X}$-ray beam used for the scanning nanodiffraction experiments of $300 \times 500 \mathrm{~nm}$.

Concerning the comparison between experimental and simulated three-dimensional RSMs whose projections on the $Q_{x} Q_{y}$ plane are shown in Figs. 4 and 11, we noticed that in general they exhibit similar peak splitting, and the evolution of peak intensities during crystal scanning is qualitatively comparable. However, the experimental and theoretical peaks have slightly different shapes and sometimes their positions in reciprocal space differ as well. We believe that this discrepancy between experiment and simulations can mainly be explained by the fact that our simulations do not consider the misfit and threading dislocations close to the $\mathrm{Si}-\mathrm{Ge}$ interface. In particular, the $1.2 \mu \mathrm{m}$ tall crystal has the highest density of threading dislocations per volume owing to its small height. Moreover, the FEM calculations have revealed that the lattice bending is not only sensitive to the strain fields caused by the misfit and threading dislocations, but also strongly influenced by the crystal morphology close to the $\mathrm{Si}-\mathrm{Ge}$ interface. Furthermore, the dislocations close to the $\mathrm{Si}-\mathrm{Ge}$ interface generate a random net tilt in every individual crystal (Falub et

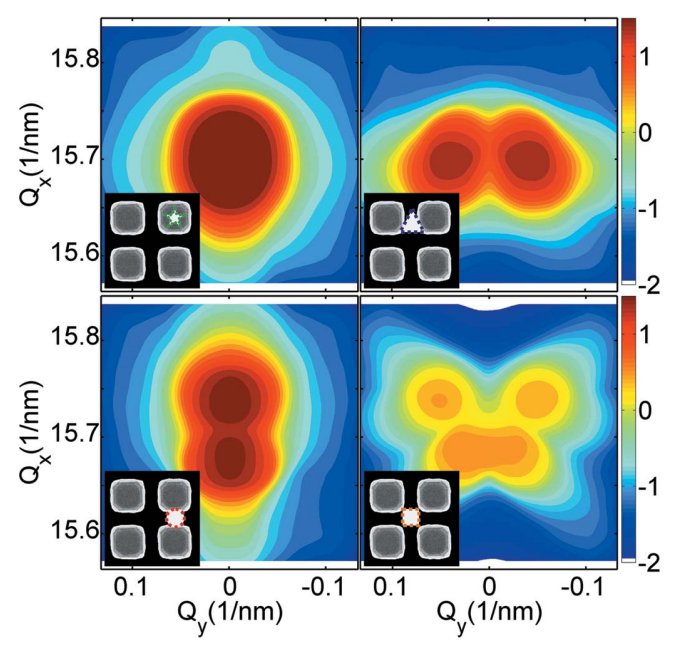

Figure 13

Simulated $Q_{x} Q_{y}$ projections of the intensity around the $\operatorname{Ge}(115)$ reflection, obtained by the convolution of the assumed disc-shaped instrumental resolution function and calculated three-dimensional RSMs shown in Fig. 11 for the $1.2 \mu \mathrm{m}$ tall crystal at four different locations of the theoretical X-ray beam, depicted by white symbols in the SEM insets. al., 2013). This tilt was not included in our calculations and can cause shifts or fluctuations in the experimental sawtooth function of Fig. 5 .

Another possible reason for the discrepancy between simulations and experiment is that the shape of the measured RSMs is also influenced by the instrumental resolution function, which is mainly determined by the beam focusing optics. Thus, all experimental data are in fact convoluted with a disc perpendicular to the beam direction, having a diameter corresponding to the beam divergence (Meduňa et al., 2014). Therefore, the experimental RSMs do not reveal all the features observed in the calculated RSMs. An example of a calculation convoluted with an expected beam shape is demonstrated in Fig. 13. In view of all these factors (dislocations, random net tilts, resolution function), we consider that the agreement between simulations and experiment is extremely good, since all the main characteristics of the intensity maps during scanning of the Ge crystals by the focused X-ray beam are very well reproduced by our threedimensional FEM model.

\section{Conclusions}

In this work the nanodiffraction process on densely spaced three-dimensional Ge crystals grown epitaxially on patterned $\mathrm{Si}$ substrates has been modelled and compared with data obtained by the scanning X-ray diffraction microscopy technique. In particular, we have simulated three-dimensional $\mathrm{X}$-ray reciprocal space maps based on finite element method data at various mesh points across individual 1.2, 3.1 and $7.8 \mu \mathrm{m}$ tall crystals. Realistic geometries of the beam and crystal shapes were used for the calculations in order to reproduce the experimental resolution of scanning nanodiffraction in real and reciprocal space. The simulations of the three-dimensional distribution of the scattered intensity on the nanoscale, based on inputs from the FEM data, allowed us to compare the local curvature of lattice planes and strain at various positions inside the Ge crystals both theoretically and experimentally. The simulated three-dimensional RSMs were found to agree well with the measured RSMs. From this we conclude that the average lattice bending diminishes with increasing crystal height, and at the top of tall crystals the bending can be neglected. Also, the strain is negligibly small far from the bottom interface, which makes these arrays of microcrystals suitable for device applications.

\section{Acknowledgements}

This work was supported by the Sinergia project NOVIPIX CRSII2_147639 and the Nano-Tera project NEXRAY of the Swiss National Science Foundation. MM wishes to acknowledge the projects INGO LG 13058, NANOE-EE2.3.20.0027 and ED1.1.00/02.0068. This research was also financially supported by the MEYS of the Czech Republic under the project CEITEC 2020 (LQ1601). We acknowledge the staff of the ID01 beamline at the ESRF, Grenoble, the Centre Suisse d'Electronique et Microtechnique (CSEM) for supplying the 
patterned Si substrates, the FIRST Center for Micro- and Nanoscience of ETH Zürich, and EMEZ electron microscopy ETH Zürich. We are also grateful for helpful discussions with B. Batlogg.

\section{References}

Bartosik, M., Daniel, R., Mitterer, C., Matko, I., Burghammer, M., Mayrhofer, P. \& Keckes, J. (2013). Thin Solid Films, 542, 1-4.

Bergamaschini, R., Isa, F., Falub, C. V., Niedermann, P., Müller, E., Isella, G., von Känel, H. \& Miglio, L. (2013). Surf. Sci. Rep. 68, 390417.

Biermanns, A., Carbone, D., Breuer, S., Jacques, V. L. R., Schulli, T., Geelhaar, L. \& Pietsch, U. (2013). Phys. Status Solidi Rapid Res. Lett. 7, 860-863.

Caha, O., Bernátová, S., Meduňa, M., Svoboda, M. \& Buršík, J. (2011). Phys. Status Solidi A, 208, 2587-2590.

Chahine, G. A., Richard, M.-I., Homs-Regojo, R. A., Tran-Caliste, T. N., Carbone, D., Jacques, V. L. R., Grifone, R., Boesecke, P., Katzer, J., Costina, I., Djazouli, H., Schroeder, T. \& Schülli, T. U. (2014). J. Appl. Cryst. 47, 762-769.

Diaz, A., Mocuta, C., Stangl, J., Vila-Comamala, J., David, C., Metzger, T. H. \& Bauer, G. (2009). Phys. Status Solidi A, 206, 18291832.

Etzelstorfer, T., Süess, M. J., Schiefler, G. L., Jacques, V. L. R., Carbone, D., Chrastina, D., Isella, G., Spolenak, R., Stangl, J., Sigg, H. \& Diaz, A. (2014). J. Synchrotron Rad. 21, 111-118.

Falub, C. V. et al. (2014). Thin Solid Films, 557, 42-49.

Falub, C. V., von Känel, H., Isa, F., Bergamaschini, R., Marzegalli, A., Chrastina, D., Isella, G., Müller, E., Niedermann, P. \& Miglio, L. (2012). Science, 335, 1330-1334.

Falub, C. V., Meduňa, M., Chrastina, D., Isa, F., Marzegalli, A., Kreiliger, T., Taboada, A. G., Isella, G., Miglio, L., Dommann, A. \& von Känel, H. (2013). Sci. Rep. 3, 2276.

Fitzgerald, E. A., Xie, Y., Green, M. L., Brasen, D., Kortan, A. R., Michel, J., Mii, Y. \& Weir, B. E. (1991). Appl. Phys. Lett. 59, 811813.

Hanke, M., Dubslaff, M., Schmidbauer, M., Boeck, T., Schöder, S., Burghammer, M., Riekel, C., Patommel, J. \& Schroer, C. G. (2008). Appl. Phys. Lett. 92, 193109.

Holý, V., Pietsch, U. \& Baumbach, T. (1999). High-Resolution X-ray Scattering from Thin Films and Multilayers. Heidelberg: Springer. Hrauda, N. et al. (2011). Nano Lett. 11, 2875-2880.

Isa, F., Chèze, C., Siekacz, M., Hauswald, C., Lähnemann, J., Fernández-Garrido, S., Kreiliger, T., Ramsteiner, M., Dasilva, Y. A. R., Brandt, O., Isella, G., Erni, R., Calarco, R., Riechert, H. \& Miglio, L. (2015). Cryst. Growth Des. 15, 4886-4892.

Isa, F., Pezzoli, F., Isella, G., Meduňa, M., Falub, C. V., Müller, E., Kreiliger, T., Taboada, A. G., von Känel, H. \& Miglio, L. (2015). Semicond. Sci. Technol. 30, 105001.

Isa, F. et al. (2016). Adv. Mater. 28, 884-888.
Kaganer, V. M., Köhler, R., Schmidbauer, M., Opitz, R. \& Jenichen, B. (1997). Phys. Rev. B, 55, 1793-1810.

Kaganer, V. M. \& Sabelfeld, K. K. (2011). Phys. Status Solidi A, 208, 2563-2566.

Känel, H. von (2012). Europhys. News, 43, 18-21.

Kent, J. P. \& Prasad, J. (2008). Proceedings of the 2008 IEEE Custom Integrated Circuits Conference, San Jose, California, USA, 21-24 September 2008, pp. 395-402.

Malachias, A., Metzger, T., Stoffel, M., Schmidt, O. \& Holý, V. (2007). Thin Solid Films, 515, 5587-5592.

Marzegalli, A., Isa, F., Groiss, H., Müller, E., Falub, C. V., Taboada, A. G., Niedermann, P., Isella, G., Schäffler, F., Montalenti, F., von Känel, H. \& Miglio, L. (2013). Adv. Mater. 25, 4408-4412.

Matthews, J. W., Mader, S. \& Light, T. B. (1970). J. Appl. Phys. 41, 3800-3805.

Meduňa, M., Caha, O., Keplinger, M., Stangl, J., Bauer, G., Mussler, G. \& Grützmacher, D. (2009). Phys. Status Solidi A, 206, 1775-1779.

Meduňa, M., Falub, C. V., Isa, F., Chrastina, D., Kreiliger, T., Isella, G. \& von Känel, H. (2014). J. Appl. Cryst. 47, 2030-2037.

Meduňa, M., Novák, J., Falub, C. V., Chen, G., Bauer, G., Tsujino, S., Grützmacher, D., Müller, E., Campidelli, Y., Kermarrec, O., Bensahel, D. \& Schell, N. (2005). J. Phys. D Appl. Phys. 38, A121-A125.

Mocuta, C., Stangl, J., Mundboth, K., Metzger, T. H., Bauer, G., Vartanyants, I. A., Schmidbauer, M. \& Boeck, T. (2008). Phys. Rev. $B, \mathbf{7 7}, 245425$.

Moore, G. E. (1965). Electronics, 38, 114-117.

Murray, C. E., Yan, H. F., Noyan, I. C., Cai, Z. \& Lai, B. (2005). J. Appl. Phys. 98, 013504.

Novák, J., Holý, V., Stangl, J., Fromherz, T., Zhong, Z., Chen, G., Bauer, G. \& Struth, B. (2005). J. Appl. Phys. 98, 073517.

Ozguven, N. \& McIntyre, P. C. (2008). Appl. Phys. Lett. 92, 181907.

Paci, B., Bailo, D., Albertini, V. R., Wright, J., Ferrero, C., Spyropoulos, G. D., Stratakis, E. \& Kymakis, E. (2013). Adv. Mater. 25, 4760-4765.

Rosenblad, C., Deller, H., Graf, T., Müller, E. \& von Känel, H. (1998). J. Cryst. Growth, 188, 125-130.

Rozbořil, J., Meduňa, M., Falub, C. V., Isa, F. \& von Känel, H. (2016). Phys. Status Solidi A, 213, 463-469.

Schmidt, O. G., Denker, U., Christiansen, S. \& Ernst, F. (2002). Appl. Phys. Lett. 81, 2614.

Stangl, J., Mocuta, C., Chamard, V. \& Carbone, D. (2014). Nanobeam $X$-ray Scattering: Probing Matter at the Nanoscale. Weinheim: Wiley-VCH Verlag GmbH.

Stangl, J., Mocuta, C., Diaz, A., Metzger, T. H. \& Bauer, G. (2009). Chem. Phys. Chem. 10, 2923-2930.

Stefenelli, M., Todt, J., Riedl, A., Ecker, W., Müller, T., Daniel, R., Burghammer, M. \& Keckes, J. (2013). J. Appl. Cryst. 46, 1378-1385.

Taboada, A. G. et al. (2014). Appl. Phys. Lett. 104, 022112.

Taboada, A. G., Meduňa, M., Salvalaglio, M., Isa, F., Kreiliger, T., Falub, C. V., Barthazy Meier, E., Müller, E., Miglio, L., Isella, G. \& von Känel, H. (2016). J. Appl. Phys. 119, 055301. 